\title{
Cognitive Impairment, Sexual Activity and Physical Tenderness in Community-Dwelling Older Adults: A Cross-Sectional Exploration
}

\author{
Rosanne Freak-Polia, Silvan Licher ${ }^{a} \quad J^{a}$ Sonne Ryan ${ }^{b} \quad$ M. Arfan Ikramª \\ Henning Tiemeier ${ }^{a, c, d}$

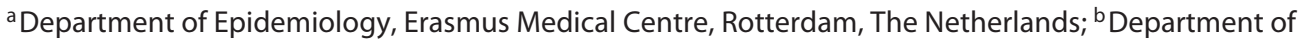 \\ Epidemiology and Preventive Medicine, Monash University, Melbourne, VIC, Australia; ' Department of Child and \\ Adolescent Psychiatry, Erasmus Medical Centre, Rotterdam, The Netherlands; ${ }^{d}$ Department of Psychiatry, Erasmus \\ Medical Centre, Rotterdam, The Netherlands
}

\author{
Keywords \\ Sexual behaviour · Touch · Geriatrics · Aged · Aging · Sex \\ factors $\cdot$ Sexual partners $\cdot$ Sexuality
}

\begin{abstract}
Background: The ability to engage in sexual activity and better cognitive functioning are both associated with better health. However, the association between cognitive functioning and sexual activity is understudied. Objective: To examine the association between cognitive functioning with sexual activity and physical tenderness among communitydwelling older adults. Methods: From the Rotterdam Study, cognitive impairment and sexual activity were assessed in 4,201 community-dwelling, 60+ year olds between 2008 and 2014 in the Netherlands. Mild cognitive impairment ( $\mathrm{MCl})$ was based upon subjective complaints related to age and education-adjusted test scores. Mini-Mental State Examination (MMSE) impairment was defined by a score of $<26$. Sexual activity and physical tenderness (e.g., fondling or kissing) in the last 6 months were assessed at an interview. Analyses
\end{abstract}

\begin{tabular}{ll}
\hline KARGER & $\begin{array}{l}\text { (c) } 2018 \text { The Author(s) } \\
\text { Published by S. Karger AG, Basel }\end{array}$ \\
E-Mail karger@karger.com & This article is licensed under the Creative Commons Attribution- \\
www.karger.com/ger & $\begin{array}{l}\text { NonCommercial-NoDerivatives 4.0 International License (CC BY- } \\
\text { NC-ND) (http://www.karger.com/Services/OpenAccessicense). } \\
\text { Usage and distribution for commercial purposes as well as any dis- } \\
\text { tribution of modified material requires written permission. }\end{array}$
\end{tabular}

were stratified by gender and partner status, with prevalence rates for the "no impairment" categories weighted based on age from the cognitive impairment categories. Inter-rater reliability was examined utilising 74 cohabiting couples of opposite gender. Results: It was found that $14 \%$ were categorised as having cognitive impairment, and $<1 \%$ as dementia (excluded from subsequent analyses). There was strong evidence that the odds of engaging in physical tenderness (observed through MMSE <26, OR 2.14, 95\% CI $1.32-3.48, p=0.002)$ and sexual activity (MCl, OR 2.36, 95\% Cl 1.35-4.12, $p=0.003$ ) among partnered females with no impairment was twice that observed among cognitively impaired partnered females. There was weak evidence that the odds of engaging in physical tenderness (MMSE $<26$, OR $1.59,95 \% \mathrm{Cl} 1.04-2.42, p=0.03$ ) and sexual activity (MMSE < 26 , OR 1.51, 95\% Cl 1.02-2.24, $p=0.04$ ) among partnered males with no impairment was $50 \%$ greater than observed among cognitively impaired partnered males. The associations between cognitive functioning and physical tenderness continued to remain after adjustment for physical function, diabetes, cardiovascular disease and cancer. There was 
no clear evidence of a difference between amnestic and non-amnestic $\mathrm{MCl}$ for sexual behaviour. There was moderate to substantial agreement among the coupled adults who had 1 partner categorised with $\mathrm{MCl}$. Conclusion: Having no cognitive impairment was associated with more engagement in sexual activity and physical tenderness among community-dwelling older adults. Sexuality is an important aspect of active aging and our findings illustrate a potential barrier to maintaining or instigating intimate relationships as we age. Longitudinal analyses are required to explore the direction of effect.

(C) 2018 The Author(s)

Published by S. Karger AG, Basel

Sexual activity contributes to quality of life and can be regarded as an essential element of human well-being and happiness [1-3]. The World Health Organization views sexual health as "a state of physical, emotional, mental and social well-being related to sexuality; not merely the absence of disease, dysfunction or infirmity" with sexuality as "a central aspect of being human throughout life" [4]. Hence, sexual activity is of interest beyond reproductive years and older adults are being increasingly incorporated in sexual activity research. Prior research among older adults has identified that engaging in sexual activity is generally associated with younger age $[5,6]$, male gender $[5,7]$, marriage or cohabitation $[5,7]$, greater social support [8] and socioeconomic position [5, $7,9,10]$, engagement in physical and social activities [5, $8]$, healthier behaviours $[5,8]$, and absence of physical and mental health conditions for both themselves and their partner [5-9, 11]. A previous limitation of research on sexual activity has been the concentration upon sexual dysfunction [12] or sexual intercourse [1, 7, 9, 11]; however, gradually the concept of sexual activity research has expanded to include aspects such as touching, holding, or close companionship expressed between individuals $[1,5,13-15]$. When assessing sexual activity among older adults, it is critical to acknowledge the importance of the broader aspects, as affection has been reported as more important to older married adults than sexual activity [16].

Recently, we explored sexual activity and physical tenderness among 2,374 community-dwelling older adults, including confirmation of inter-rater reliability through 304 opposite-sex couples [5]. However, we excluded individuals with cognitive impairment or dementia, which is common practice among older adult samples due to difficulties surrounding reliability of self-report ques- tionnaires and potential concerns regarding confounding and effect modification. Greater cognitive function has been associated with better physical and psychological health, and greater quality of life among older adults [17]. However, there is limited research examining the association between cognitive functioning and sexual activity in the ageing population, particularly within non-patient settings [17, 18]. A systematic review published in 2014 identified only 8 publications from 7 cohorts, of which 5 recruited dementia patients and/or their partners and only 2 recruited older persons through other sources, specifically through general practitioners or community mass mailing [18]. These cross-sectional comparisons demonstrated an "indication that sexual activity in later life is associated with better overall cognitive functioning...The few studies found are limited, methodologically weak, and inconclusive" [18]. Since this systematic review, we have identified 2 further studies assessing the association between cognitive functioning and sexual activity among older adults $[17,19,20]$.

This paper aims to contribute to our understanding of the association between cognitive functioning and sexual activity (assessed as sexual activity and physical tenderness) through the assessment of a large sample of community-dwelling, older adults aged 60 years or older. First, we provide cross-sectional prevalence of sexual activity and physical tenderness among cognitively impaired, community-dwelling older adults. Second, we compare the prevalence of sexual activity and physical tenderness between impaired and non-impaired older adults. Third, we assess the reliability of self-reported sexual activity within a sub-sample of cognitively impaired older adults and their non-cognitively impaired cohabitating partner of the opposite gender. Results were stratified by gender and partner status due to their differential effects upon sexual availability $[1,5,21]$.

\section{Methods}

\section{Study Sample}

The Rotterdam Study is a population-based cohort designed to examine the onset of disease in older adults [22]. In 1990, 7,983 residents aged $55+$ years from the district of Ommoord were recruited to the Rotterdam Study, followed by a second recruitment wave in 2000 of 3,011 residents aged 55+ years and a third recruitment wave in 2006 of 3,932 residents aged $45+$ years. As our study population was recruited from 1 suburb in a country with relatively low social inequity [23], it can be considered homogenous, particularly in terms of socioeconomic position. While the original recruitment cohort had prominently Dutch heritage, the district had changed and a range of ethnic backgrounds prominent in the 
Netherlands were recruited for participation in the second and third cohorts. The Rotterdam Study was approved by the medical Ethics Committee according to the Population Study Act Rotterdam Study, executed by the Ministry of Health, Welfare and Sports of the Netherlands. All participants gave written informed consent. All questions were asked face-to-face at the Rotterdam Study data-collection facility or at the participant's home. All questions had pre-determined answer options.

This paper is a secondary analysis of the 5,948 participants aged 60 years or more (aged 60-106 years, mean 73.2 \pm 8.1 SD, born between December 1904 and February 1954), who attended the data collection round from December 2008 to May 2014. As part of the Rotterdam Study, they were first asked questions based on their sexual activity and physical tenderness (Appendix 1). Two of the trained interviewers felt uncomfortable asking these sexual activity and physical tenderness questions; one asked $0 \%$ (out of 479) and the other asked $31.62 \%$ (out of 136) of their allotted participants, and the 43 participants were excluded from analysis. The remaining 18 interviewers asked $80.46 \%$ (range $64.11-100 \%, n=$ 4,291 ) of participants the sexual activity and physical tenderness questions. Participants were less likely to be asked sexual activity questions by the trained interviewers if they had dementia, lower functional ability, and lived in a nursing home (Appendix 2). There was no difference in regard to being asked sexual activity questions between living independently and in a serviced flat (data not reported, $p=0.5$ ). Adjustment by age or gender did not alter these associations.

Of the 4,291 participants who were asked the sexual activity and/or physical tenderness questions, 4,273 had cognitive impairment information. Participants were excluded if they answered "I do not know" to both sexual activity and physical tenderness questions $(n=40)$, had incomplete partner status $(n=5)$, lived in a nursing home $(n=12)$ or did not provide consent for data linkage $(n=15)$. The final sample consisted of 4,201 older adults. The 72 excluded participants were similar to the 4,201 included participants in terms of age $(p=0.9)$, gender $(p=0.9)$, partner status $(p=$ $0.5)$, education $(p=0.5)$, sexual activity $(p=0.9)$ and physical tenderness engagement $(p=0.3)$.

\section{Sexual Activity and Physical Tenderness}

Participants were assessed by trained interviewers in face-toface interviews either at home or at the Ommoord district research centre and were asked "Have you been sexually active in the past 6 months?" with response options "yes," "no" or "I do not know." If the response was "no," participants were subsequently asked "Have you experienced other forms of physical tenderness in the last 6 months (e.g., fondling or kissing)?”.

\section{Mild Cognitive Impairment}

Mild Cognitive Impairment (MCI) was defined as the presence of subjective and objective cognitive complaints in the absence of dementia for Rotterdam Study participants aged 60 years or more using official criteria, and has been described in detail elsewhere [24]. Subjective cognitive complaints were examined by answering "yes" to at least 1 question evaluating memory ( 3 items: difficulty remembering, forgetting what 1 had planned to do, and difficulty finding words) or everyday functioning ( 3 items: difficulty managing finances, problems using a telephone, and difficulty getting dressed) [24]. Objective cognitive impairment was derived from a cognitive test battery comprising letter-digit substitution task,
Stroop test, verbal fluency test and 15-word verbal learning test based on Rey's recall of words. To obtain more robust measures, compound scores for various cognitive domains including memory function, information-processing speed and executive function were constructed [24]. Compound scores for memory, information processing speed and executive function were calculated using Z-scores, and a person was classified as cognitively impaired if they scored below 1.5 SD of the age and education adjusted means of the study population. For MCI subtypes, the distinction by Roberts and Knopman et al. [25] was followed. As described by de Bruijn et al. [24], "Amnestic MCI was defined as persons with MCI who had an impaired test score on memory function (irrespective of other domains). Non-amnestic MCI was defined as persons with MCI having normal memory function, but an impaired test score on executive function or information-processing speed."

\section{Cognitive Impairment Based on Mini Mental}

\section{State Examination}

Cognitive impairment was defined as a Mini Mental State Examination (MMSE) score of less than $26(<26)$. For the Rotterdam Study, an MMSE score $<26$ has been previously utilised to distinguish cognitive impairment, and has been utilised as an exclusion criteria when completing self-reported questionnaires [5, 21, 26]. The MMSE is a validated, self-reported instrument that measures general cognitive function. It consists of 30 questions examining orientation, registration (repeating named prompts), attention and calculation, recall, language and ability to follow simple commands.

\section{Dementia}

Participants were screened for dementia at baseline and subsequent Rotterdam Study centre visits with the MMSE and the Geriatric Mental Schedule [30, 32] organic level [27]. Those with an MMSE $<26$ or Geriatric Mental Schedule score $>0$ underwent further examination and informant interview, including the Cambridge Examination for Mental Disorders of the Elderly. In addition, all participants underwent routine cognitive assessment and the entire cohort were continuously monitored for dementia through electronic linkage of the study database with medical records from general practitioners and the regional institute for outpatient mental health care. Available information on cognitive testing and clinical neuroimaging was used when required for diagnosis of dementia subtype. A consensus panel led by a consultant neurologist established the final diagnosis according to standard criteria for dementia (Diagnostic and Statistical Manual of Mental Disorders, Third Edition, Revised), Alzheimer's Disease (National Institute of Neurological and Communicative Diseases and Stroke/ Alzheimer's Disease and Related Disorders Association) and vascular dementia (National Institute of Neurological Disorders and Stroke and Association Internationale pour la Recherché et l'Enseignement en Neurosciences).

\section{Potential Health Confounders}

Activities of daily living [28,29] (ADL), diabetes, cardiovascular disease (CVD) and cancer were selected a priori selected [30] as potential confounders for the relation between cognitive functioning and sexual activity. Diabetes, CVD (stroke, heart failure and coronary heart disease) [31] and cancer are 3 of the 4 most common chronic disease domains [32]. Diabetes mellitus was defined as selfreported through questionnaire and/or a serum fasting glucose 
Table 1. Categorisation of cognitive impairment and dementia

\begin{tabular}{|c|c|c|c|c|c|c|}
\hline & & \multicolumn{5}{|c|}{ MMSE cognitive impairment } \\
\hline & & $\begin{array}{l}\text { none } \\
(\text { MMSE } \geq 26)\end{array}$ & $\begin{array}{l}\text { impairment } \\
(\mathrm{MMSE}<26)\end{array}$ & dementia & missing & total \\
\hline \multirow[t]{4}{*}{ MCI } & No MCI & 3,425 & 294 & 0 & 158 & 3,877 \\
\hline & MCI & 198 & 98 & 0 & 0 & 296 \\
\hline & Dementia & 0 & 0 & 28 & 0 & 28 \\
\hline & Total & 3,623 & 392 & 28 & 158 & 4,201 \\
\hline
\end{tabular}

MCI, mild cognitive impairment; MMSE, mini mental state examination.

measurement of $\geq 7.0 \mathrm{mmol} / \mathrm{L}$ using laboratory data derived from blood sampling performed at the research centre. For CVD and cancer, incident data was obtained through continuously monitoring day-to-day medical records and coded with agreement from 2 research physicians [31,33] and was available up to April 1, 2010.

\section{Stratification and Weighting}

Results were stratified by gender (male or female) and partner status (partnered or unpartnered) due to their differential effects upon sexual availability $[1,5,21]$ and cognitive impairment. Partner status was determined by asking (1) "What is your marital status?" with response options "never been married," "married/ cohabiting," "widowed" and "divorced." Those who did not answer "married/cohabiting" were asked (2) "Do you currently have a partner?" with response options "yes, a partner with whom I live," "yes, a partner with whom I do not live" and "no, I have no partner." Those who answered "married/cohabiting," "yes, a partner with whom I live," or "yes, a partner with whom I do not live" were considered "partnered," while those who answered "no, I have no partner" to the second question were considered "unpartnered."

Increasing age is a strong predictor of reduced engagement in sexual activity $[5,6]$ and increased cognitive impairment. To provide comparative sexual activity and physical tenderness rates by cognitive status, the "no impairment" groups were weighted based upon 5-year age bands from the "cognitive impairment" groups after partner and gender stratification using the fweight command in Stata. There were eight 5 -year age bands starting at $60<65$, and ending with $100 \leq 106$.

\section{Statistical Analysis}

First, we categorised participants into cognitive impairment categories of "dementia," "MCI" or "no MCI," and "MMSE <26" or "MMSE $\geq 26$," and examined demographic differences between categories. Second, we assessed the prevalence of sexual activity and physical tenderness in the previous 6 months stratified by cognitive impairment category, gender and partner status groups.

Through sensitivity analyses we explored the second aim by adjustment of potential health confounders, by further categorisation of amnestic and non-amnestic sub-groups for MCI and by assessment in a sub-sample of couples who cohabited at the same address, were of the opposite gender and 1 partner was categorised as MCI. We had no reliable information on same-sex couples.
Cross-sectional binary logistic regression analyses were performed using Stata version 14 . We defined statistically significance as a $p$ value of $<0.05$ and results are interpreted in accordance with Wasserstein and Lazar [34].

\section{Results}

\section{Cognitive Impairment Status}

One in seven participants was categorised as having cognitive impairment or dementia, Table 1. Slightly more participants were categorised as having cognitive impairment through the MMSE (9.33\%) than the MCI $(7.05 \%)$, and less than $1 \%$ were categorised as having dementia, Table 1. As expected, participants categorised as "no impairment" were more likely to be younger, be partnered, be highly educated, have greater physical functioning, have no diabetes and have no cardiovascular disease than participants with cognitive impairment, Table 2. Cognitive impairment categorisation was distinctly different between MMSE and MCI ( $\mathrm{chi}^{2}=198$, $p<0.001$ ), Table 1 . There were 158 participants with a missing MMSE score, who had other measures to categorise them as having no MCI. Participants with dementia were more likely to be older than participants with no impairment and participants with MCI. The number of participants categorised as having dementia $(n=28)$ was too small for further exploration and presented demographic differences should be interpreted with caution.

\section{Cognitive Impairment Status, Sexual Activity and \\ Physical Tenderness}

In general, being categorised as having cognitive impairment was associated with lower engagement in sexual activity and physical tenderness; however, the strength of the association varied by cognitive impairment mea- 


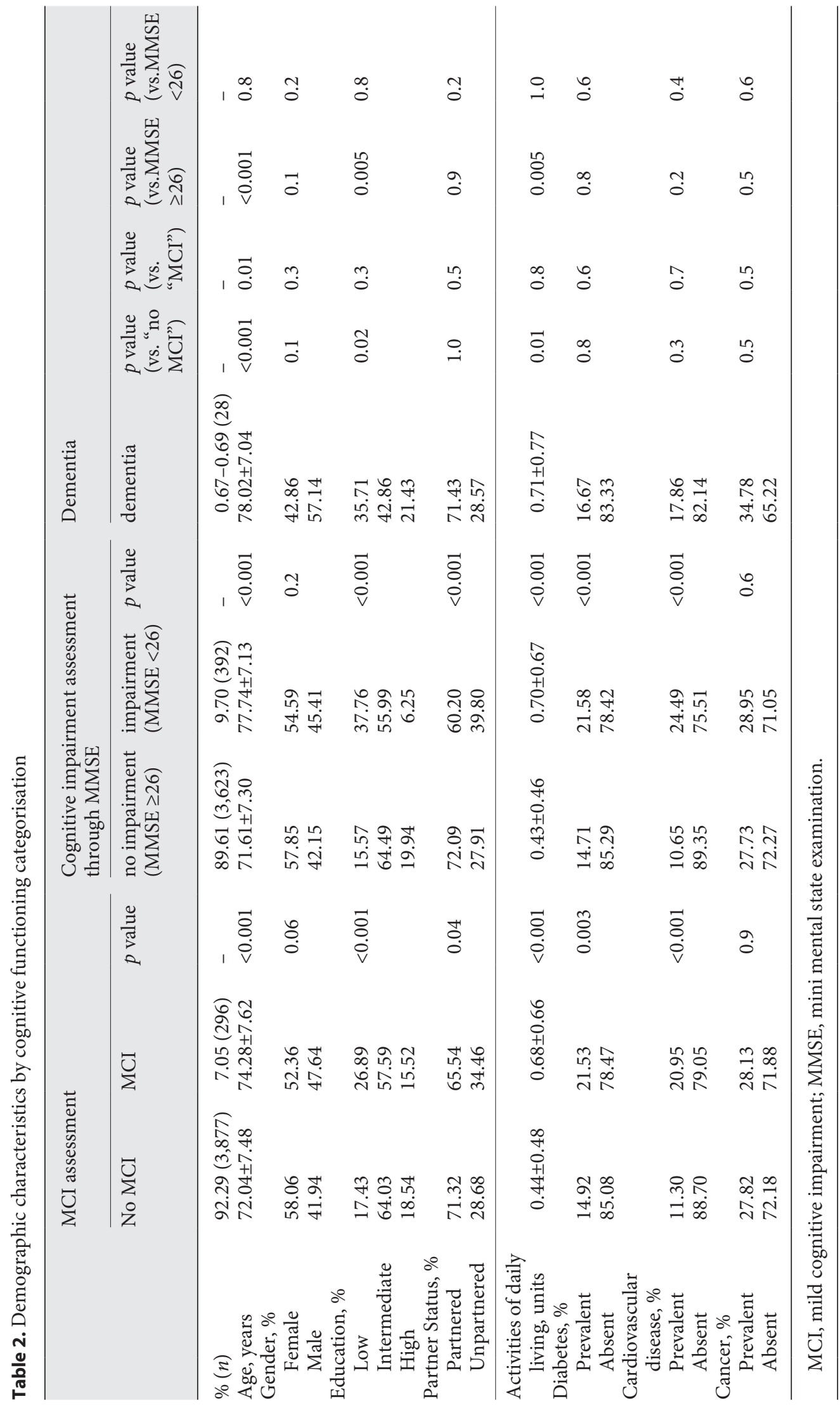




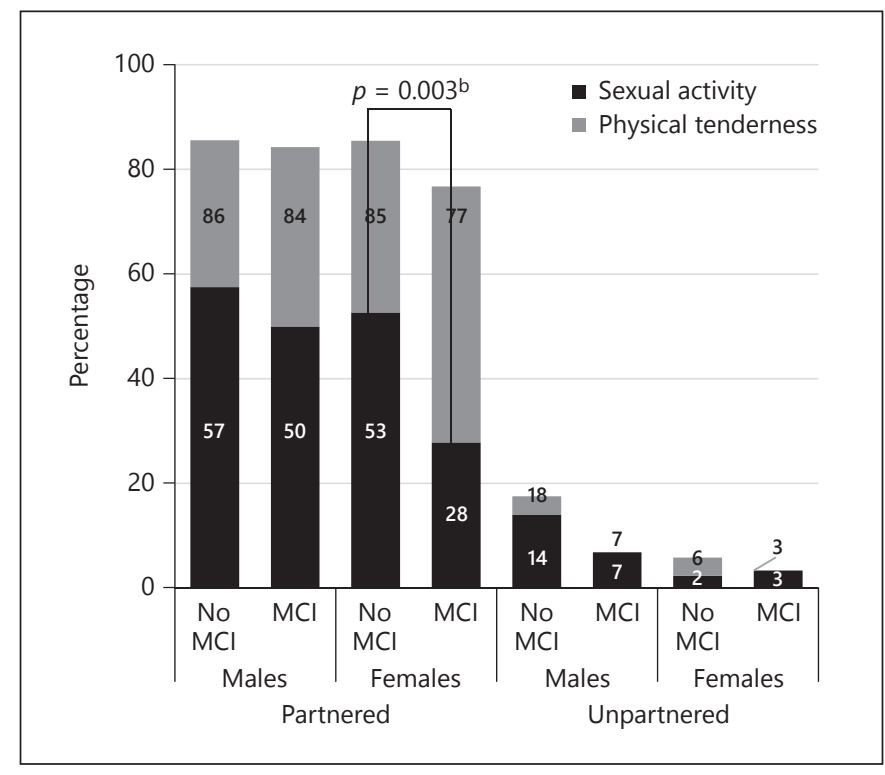

Fig. 1. Percentage of older adults engaging in sexual activity or physical tenderness in the previous 6 months, weighted by mild cognitive impairment ${ }^{\mathrm{a}}$. (MCI) 5 year age category. ${ }^{\mathrm{a}}$ The number of participants categorised as having dementia $(n=28)$ was too small for further exploration and have been excluded. ${ }^{\mathrm{b}}$ Comparing sexual activity among partnered females with or without MCI.

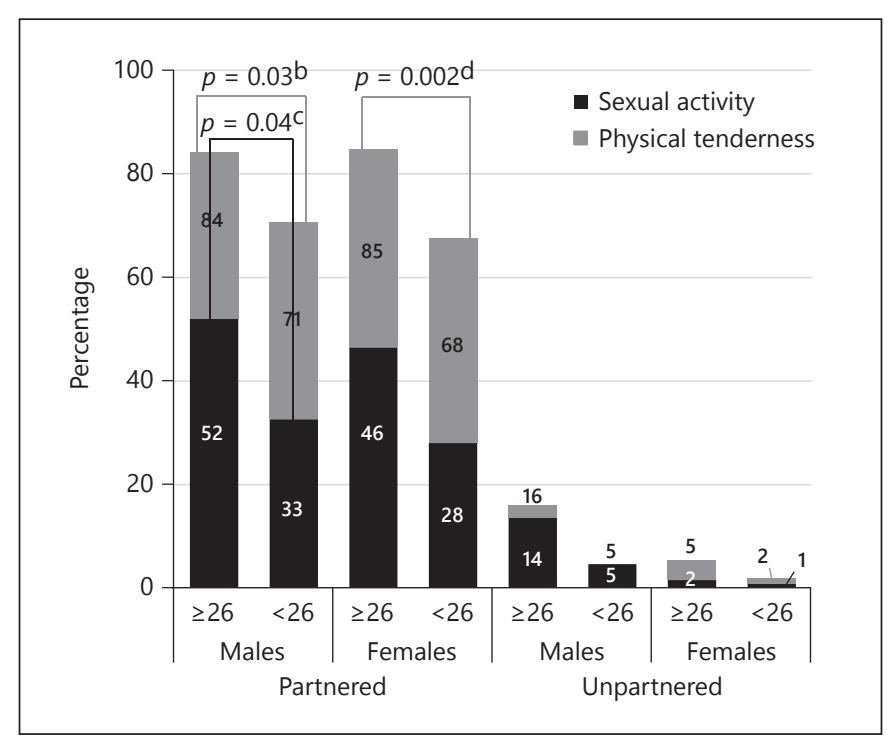

Fig. 2. Percentage of older adults engaging in sexual activity or physical tenderness in the previous 6 months, weighted by Mini Mental State Examination cognitive impairment ${ }^{\mathrm{a}}(\mathrm{MMSE}<26) 5$ year age category. ${ }^{\text {a }}$ The number of participants categorised as having dementia $(n=28)$ was too small for further exploration and have been excluded. ${ }^{b}$ Comparing physical tenderness among partnered males with or without MCI. ${ }^{c}$ Comparing sexual activity among partnered males with or without MCI. ${ }^{\mathrm{d}}$ Comparing physical tenderness among partnered females with or without MCI. surement (Fig. 1, 2). Low sexual activity and physical tenderness engagement among unpartnered participants restricted further exploration. When categorised by MCI, there was strong evidence that the odds of engaging in sexual activity among partnered females with no impairment was at least twice that observed among partnered females with MCI (OR 2.36, 95\% CI 1.35-4.12, $p=0.003$, Appendix 3). However, there was no evidence of an association between MCI impairment and physical tenderness or among males for sexual activity $(p>0.05$, results not shown).

When MMSE was assessed as a continuous measure (mean $27.77 \pm 2.34 \mathrm{SD}$ ), each unit increase was associated with a $0.60 \%$ higher likelihood of engaging in sexual activity $(p=0.004)$ and a $0.70 \%$ higher likelihood of engaging in physical tenderness $(p<0.001)$, after adjustment for age, gender and partner status. When categorised by the MMSE cut-off of 26, there was weak evidence that the odds of engaging in sexual activity (OR $1.51,95 \%$ CI $1.02-2.24, p=0.04$, Appendix 3 ) and physical tenderness (OR 1.59, 95\% CI 1.04-2.42, $p=0.03$ ) was at least $50 \%$ greater among partnered males with no impairment than that observed among partnered males with impairment. There was also strong evidence that the odds of engaging in physical tenderness among partnered females with no impairment based on MMSE was at least twice that observed among partnered females with MMSE impairment (OR 2.14, 95\% CI 1.32-3.48, $p=$ 0.002 ).

\section{Adjustment for Potential Health Confounders}

After adjustment for ADLs, prevalent diabetes, CVD and cancer, the association between MMSE categorisation and physical tenderness continued to remain, and the magnitude of the association increased for males (Appendix 3). After adjustment, the magnitudes reduced and there was no longer evidence of an association between sexual activity with MCI categorisation for females and sexual activity with MMSE categorisation for males.

\section{Amnestic and Non-Amnestic Sub-Groups of MCI}

When compared to no impairment, there was no clear evidence of a difference compared to no MCI between with-amnestic $(n=104)$ and non-amnestic $(n=$ 192) sub-groups of MCI in terms of sexual activity (adjusted for age, sex and partner status: $p=0.06$ and $p=0.6$, respectively) or physical tenderness ( $p=0.8, p=0.09$ ). When amnestic was compared to non-amnestic, there was no evidence of a difference in terms of sexual activity 


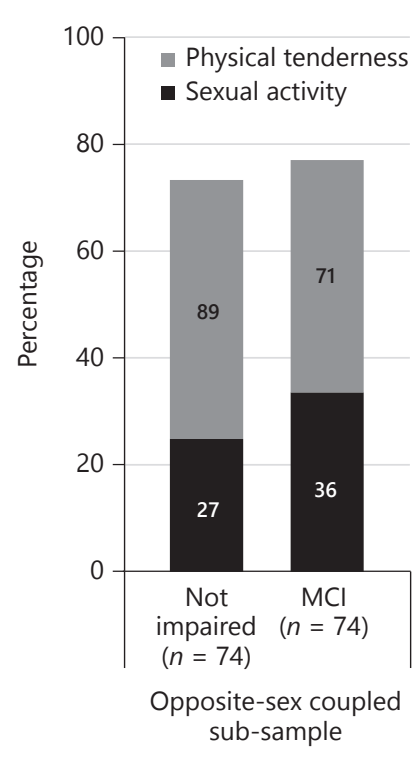

Fig. 3. Percentage of opposite-sex coupled cohabiting older adults ${ }^{\mathrm{a}}$ engaging sexual activity or physical tenderness in the previous 6 months, comparison by mild cognitive impairment ${ }^{\mathrm{b}}$ (MCI) status $(n=74) .{ }^{\text {a }}$ Opposite-sex cohabiting partnered adults with only one partner categorised as having MCI. ${ }^{b}$ The number of participants categorised as having dementia $(n=28)$ was too small for further exploration and have been excluded.

( $p=0.4$ ) or physical tenderness $(p=0.6)$. No difference between amnestic and non-amnestic sub-groups of MCI with sexual activity or physical tenderness was also observed when further adjusted for ADLs, prevalent diabetes, CVD and cancer (data not shown).

\section{Coupled Sub-Sample}

We identified 79 couples with at least 1 partner categorised as having MCI (mean age $75.57 \pm 5.88$ SD years; men: $76.60 \pm 5.7$, women: $74.54 \pm 5.96$ ). However, 5 couples were excluded prior to analysis; 3 couples had both partners categorised as having MCI (partners agreed: no sexual activity engagement and 1 couple engaged in physical tenderness) and 2 couples did not respond to the sexual activity question ( 1 couple agreed that they engaged in physical tenderness, the other disagreed). There was substantial similarity between partners for their self-reported sexual activity (90.5\% in agreeance, Kappa 0.784, 95\% CI 0.56-1.01) and moderate agreement for physical tenderness $(90.7 \%$ in agreeance, Kappa 0.664, 95\% CI 0.44-0.89; Fig. 3). Patterns of agreement have also been observed in a prior sub-sample from this study population [5]. If the woman was the partner with
MCI, there was slightly more agreeance $(n$ couples $=24$, sexual activity: $95.8 \%$, kappa $0.909,95 \%$ CI $0.51-1.31$ ) than if the man was the partner with MCI ( $n$ couples = 50, sexual activity: $88.0 \%$ agreeance, kappa $0.719,95 \%$ CI $0.45-0.99)$.

\section{Discussion}

In our study population, 1 in 7 community-dwelling older adults had cognitive impairment or dementia. Less than $1 \%$ were categorised as having dementia and were excluded from subsequent analyses. In general, cognitive impairment was associated with lower engagement in sexual activity and physical tenderness; however, the strength of the association varied by cognitive impairment measurement. For partnered older adults, there was strong evidence that the odds of engaging in physical tenderness (observed through MMSE <26) and sexual activity (MCI) among females with no impairment were at least twice that observed among cognitively impaired females. There was weak evidence that the odds of engaging in physical tenderness (MMSE <26) and sexual activity (MMSE <26) among males with no impairment were at least $50 \%$ greater than that observed among cognitively impaired males. After adjustment for ADLs, diabetes, CVD and cancer, the association between no impairment and greater sexual activity no longer remained. There were no differences between amnestic and non-amnestic sub-groups of MCI. There was agreeance for both sexual activity and physical tenderness between the coupled subsample that had 1 partner categorised as having MCI.

Our cognition and sexual activity findings are in line with the findings of 3 comparable cross-sectional studies among older adults, particularly as we also observed that the strength and magnitude of the association with sexual activity varied by cognitive impairment measurement. Among 335 Italians aged over 65 years, who were recruited through general practitioners, sexual activity questions were answered between 1992 and 1995 [35]. While there was limited evidence that self-reported cognitive functioning examined through 5 questions (ability to concentrate, feelings of confusion and any mnesic difficulties) was associated with sexual interest or sexual activity, there was moderate evidence that MMSE was associated for sexual interest (OR 2.13, $p=$ 0.01 ) and sexual activity (OR 2.32, $p=0.01$ ) [35]. The 335 person sample had greater MMSE cognitive impairment (mean score $25.3 \pm 4.1$ [35]) than our sample, with 56\% [36] having an MMSE $\leq 26$ compared to $20 \%$ 
in our sample (however, we utilised MMSE $<26$ and reported 10\%). Among the 335 older adults with MMSE $\leq 26,12.7 \%$ reported sexual interest and $4.6 \%$ reported sexual activity, which are much lower than our comparable unadjusted sexual activity prevalence rate of $26.72 \%$ - possible due to the generational differences [20] between samples. While further exploration was undertaken $[35,36]$, the sample size limited conclusions, especially by age and gender stratification. Among 662 older community-dwelling menopausal American women aged 57-90 years, sexual satisfaction was associated with subjective attitudes towards aging and emotional functioning, rather than cognitive status [37]. However, the 662 sampled studies were presented as an abstract and the association between sexual satisfaction and cognitive status in the absence of additional adjustments was not presented; hence, these results are not comparable to our study. Among 6,833 representative, community-dwelling English aged 50-89, questions related to sexual activity was asked as part of the English Longitudinal Study of Ageing [17]. Within English Longitudinal Study of Ageing, sexual activity was defined as an activity that included "intercourse, masturbation, petting or fondling" [17], and hence could be considered a measure in-between our "sexual activity" and "physical tenderness" questions. Among the 3,060 older men, there was strong evidence of an association between sexual activity and both number sequencing and recall (mean difference after adjustment for age, education and wealth; sequencing: $4.6, p<0.001$, recall: $0.7, p<0.001)$. However, among the 3,773 women there was strong evidence of an association between sexual activity and recall only $(0.3, p<0.002$; sequencing 1.4 , $p=0.1$ ). While our findings presented in this paper are similar, the 6,833 sample included much younger adults (mean 66 years) and examined cognitive tests continuously rather than categorising cognitive impairment, which limits comparability. Among 73 communitydwelling English aged 50-83, there was weak to moderate evidence that weekly engagement in sexual activity (compared to never) was associated with cognitive functioning examined through fluency $(B-1.63 \pm 0.65$ $\mathrm{SE}, p=0.01)$ and the validated cognitive questionnaire Addenbrooke's Cognitive Examination-III (ACE-III; $B$ $-4.39 \pm 2.07 \mathrm{SE}, p=0.04$ ), after adjustment for age, gender, education and cardiovascular health [22]. A measurement of sexual frequency was certainly a strength of this 73 sampled study, especially because a dose-response association with cognitive domains was observed. However, there was no evidence of a difference between engaging weekly or monthly in sexual activity, and no evidence of the association when cognitive functioning was examined through visuospatial, attention, memory or language. The authors noted that a larger sample size may be required to detect smaller differences, which may also be relevant to our MCI findings, as they were in the same direction but generally of lower in magnitude compared to the MMSE $<26$ categorisation. Additionally, a larger sample size may be required to assess MCI subtypes. Despite differential underlying aetiology, pathology, clinical presentation and outcomes [25], there was no difference between the MCI subtypes of amnestic (impaired memory function) and non-amnestic (impaired executive function or information-processing speed) with regard to sexual activity in our sample. There are a range of cognitive measures from validated cognitive questionnaires, which can be used for diagnosis (for example MCI) to those which are commonly used for screening purposes (e.g., MMSE, number sequencing and fluency), and these are likely testing different concepts of cognitive functioning. Furthermore, some cognitive questionnaires are developed to be sensitive to cognitive decline (e.g., MMSE), rather than cognitive variation. In relation to sexual activity, the current literature generally reflects that the cognitive impairment measures used for diagnosis have a weaker and lower magnitude of association with sexual activity when compared to the cognitive impairment measures used for screening purposes.

Co-morbidity may account for some of the association between reduced sexual activity and reduced cognitive function. In our study sample, we observed that, as expected, older adults with no cognitive impairment were more likely to have greater physical functioning and lower prevalence of chronic disease. Prior research among older adults has identified that engaging in sexual activity is generally associated with the absence of physical and mental health conditions for both themselves and their partners $[5-9,11]$. Hence, our finding that the association between cognitive impairment and sexual activity no longer remained after adjustment for ADLs, diabetes, CVD and cancer is somewhat expected. It is unclear whether 2 [35-37] of the comparable cross-sectional studies adjusted analyses, although a range of physical health, psychological health, social function, life satisfaction, attitude and resilience aspects were mentioned between the 2 studies. Fully adjusted models did not affect the findings from the study of 6,833 English older adults [17]. Their first model adjusted for age, education and wealth (findings summarized above), while the final model further 
adjusted for physical activity, cohabiting and self-rated health, depression, quality of life and loneliness. However, their sexual activity question is not directly comparable as it could be considered a measure in-between our "sexual activity" and "physical tenderness" questions. Our finding that no impairment was associated with physical tenderness after co-morbidity adjustment illustrates that cognitive functioning may be a potential barrier to maintaining or instigating intimate relationships.

As the current evidence examining sexual activity and cognitive impairment is cross-sectional, we can only speculate upon the direction of effect at this time. There are theoretical explanations for the direction of effect in both directions, and it is possible that the association is bi-directional. Engaging in sexual activity is generally associated with better psychological and physical health [5], and improved cognition or prolonged cognitive function may be one benefit. A range of hormones are released during sexual activity and orgasm, including dopamine, which is associated with motor function [38]. Additionally, variations in sex-related hormones, such as higher levels of testosterone, lower estradiol, higher follicle-stimulating hormone, higher luteinising hormone, higher dehydroepiandrosterone sulfate and higher prolactin are associated with poorer cognition [39].

Alternatively, structural processes can deteriorate as part of cognitive impairment and may impact sexual activity.

Sexual problems associated with dementia are well established, with neuronal loss theorised to proceed altered sexual activity [40]. More current literature also discuss the cognitive and decision-making processes involved in sexual behaviour: "In the process of sexual functioning and reaching an orgasm, there are several identified moments in sexual behavior at which a decision is or can be made. The decision-making process involves aspects of judgment, consent, sense of self, sense of other, and abstract thinking, all of which require intact memory and executive functioning" [18]. As examples, sexual arousal involves cortical functions including the limbic and paralimbic cortexes [41], and these structures may be impacted through cognitive impairment [18]. One way in which these structural and cognitive processes may lead to the variance in sexual behaviour is through a decline in sexual interest. Cross-sectional comparisons among community-dwelling elderly identified that higher cognitive functioning was associated with perceiving sexuality as important, remaining a need for intimacy when getting older, and evaluating sexual life as pleasant [42]. Hence,

Cognitive Impairment, Sex and

Tenderness future research should not only incorporate broader aspects as affection but also incorporate aspects of sexual interest.

\section{Strengths and Limitations}

The main limitation of this study is the low number of participants with dementia, which is likely to be an under-representation due to the interviewers being more likely to ask sexual activity questions to participants without dementia. Additionally we identified several common limitations of sexual activity research that were also present in this study. First, within the unpartnered older adults, there was low sexual activity engagement and a small sample size for men [5], and hence, we were likely underpowered to examine differences in unpartnered participants. While this limited our exploration among unpartnered older adults, there is no reason that the relation between cognitive functioning and sexual activity would be different by partner or/ and gender status. If we had a larger sample of unpartnered older adults, we hypothesize that the relation between cognitive functioning and sexual activity would be reflective of the relation within the partnered cohort. Second, there were very few older adults with dementia to examine specific associations. Third, sexual orientation (homosexual, heterosexual or bisexual) is rarely considered and was not assessed in our or the other studies. Fourth, "sexual activity" was a single question and left open for interpretation [5]. It is possible that the individual in the different groups (as defined by stratification for age, gender or cognitive impairment status) interpret the questions differently, leading to different understanding and some misclassification. It is only recently that research has incorporated broader definitions of sexual activity as any sexually arousing activities [43]. In this study, we provided the examples of fondling and kissing for physical tenderness. Nevertheless, because, inevitably, each participant interpreted the question a wide range of sexual behaviours, including solo masturbation (which is more common for older men than older women [14, $44]$ ), could have been overlooked. Future research would benefit from the frequency of sexual behaviour, additional clarification of activities such as solo masturbation, and additional questions pertaining to sexual opinions, feelings and function. Finally, this study design is cross-sectional and further longitudinal research is required.

While having 2 measures of cognitive impairment is a strength of this study, the MMSE may have floor and ceil- 
ing effects within community samples that should be considered when interpreting data from longitudinal studies of cognitive decline [45]. This paper overcomes several limitations of sexual behaviour research as we explicitly asked about physical tenderness (not just sexual activity) in older age groups though assessment in one of the largest samples of older adults [9, 20,46-48] who were not recruited explicitly to talk about their sexuality nor were limited by partner status or sexual orientation. Additional strengths include the recent interval (of 6 months rather than one year $[13,49])$, and the assessment of MCI [24]. Our results of an association between cognitive impairment and sexual behaviour are likely generalisable to community-dwelling older adults in Westernized cultures. As the Netherlands is known for being open-minded on sexual matters [50], particularly in regards to homosexuality, and has relatively low social inequity [23], prevalence rates may differ between countries with different cultural norms.

\section{Knowledge Translation}

Our findings contribute to the understanding of sexual activity in later life, and respond to the claim by older adults that their lives can be improved by normalising sexual activity and desire [51]. Here we illustrate that reductions in cognitive function form a potential barrier to maintaining or instigating intimate relationships.

Sexuality is an important aspect of active aging and is increasingly important to older adults $[52,53]$. The stereotyping of older adults as not interested or not engaging in sexual activity has direct implications for the physical health and well-being of older adults. Undiagnosed or untreated sexual problems can lead to depression, anxiety, social withdrawal and other mental health issues [54]. Addressing sexual activity among older adults and even having open discussions can contribute to maintaining and improving quality of life and reducing misconceptions. We encourage health care professionals to proactively address sexuality and extend knowledge about safe sex and sexual functions to older adults.

\section{Conclusion}

This is the first study to present prevalence rates for either sexual activity or physical tenderness among older community-dwelling adults who are cognitively impaired, and the first study to examine inter-rater reliabil- ity within a coupled sub-sample who had one partner with cognitive impairment. Our findings are aligned with prior research, and highlights that greater cognitive impairment is associated with less engagement in sexual activity and physical tenderness. Additionally, we illustrated that co-morbidity may explain the reductions in sexual activity but not physical tenderness for cognitive impaired older adults. As the strength and magnitude of the association with sexual behaviour varies by cognitive impairment measurement, future research requires thought as to the sensitivity of such cognitive measures. Additionally, there are implications for sexual behaviour research in community samples, as exclusion criteria based on cognitive functioning may provide different outcomes. Sexuality is an important aspect of active aging and our findings illustrate a potential barrier to maintaining or instigating intimate relationships as we age. As findings are cross-sectional, longitudinal analysis is required to explore the direction of effect.

\section{Acknowledgements}

We would like to thank Frank van Rooij, Renée de Bruijn, Annemarie Luik, Maarten Leening, and Hoyan Wen for their assistance with data preparation.

\section{Author Contributions}

R.F.-P. takes responsibility for the analysis design, the integrity of the data, the accuracy of the data analysis and the critical interpretation of the data. H.T. supervised data collection on sexual activity and physical tenderness, and contributed to interpretation of the results. All authors contributed to the final version of the paper and have read, as well as, approved of the final manuscript.

\section{Financial Disclosures}

The Rotterdam Study is supported by Erasmus Medical Centre and Erasmus University Rotterdam, the Netherlands Organization for Scientific Research (NWO), the Netherlands Organization for Health Research and Development (ZonMw), the Netherlands Genomics Initiative, the Ministry of Education, Culture and Science, the Ministry of Health, Welfare and Sports and the European Commission (DG XII). RFP, GDCL, AH and HT are affiliated with Erasmus Medical Centre. RFP is also affiliated with Monash University. RFP is supported by a NHMRC ECR Fellowship (1053666). The authors declare no further conflicts of interest. The data collection, analysis and interpretation of data; the writing of the manuscript; and the decision to submit the manuscript for publication were solely at the discretion of the Erasmus researchers, independent of the funders. 


\section{Appendix 1}

Flowchart

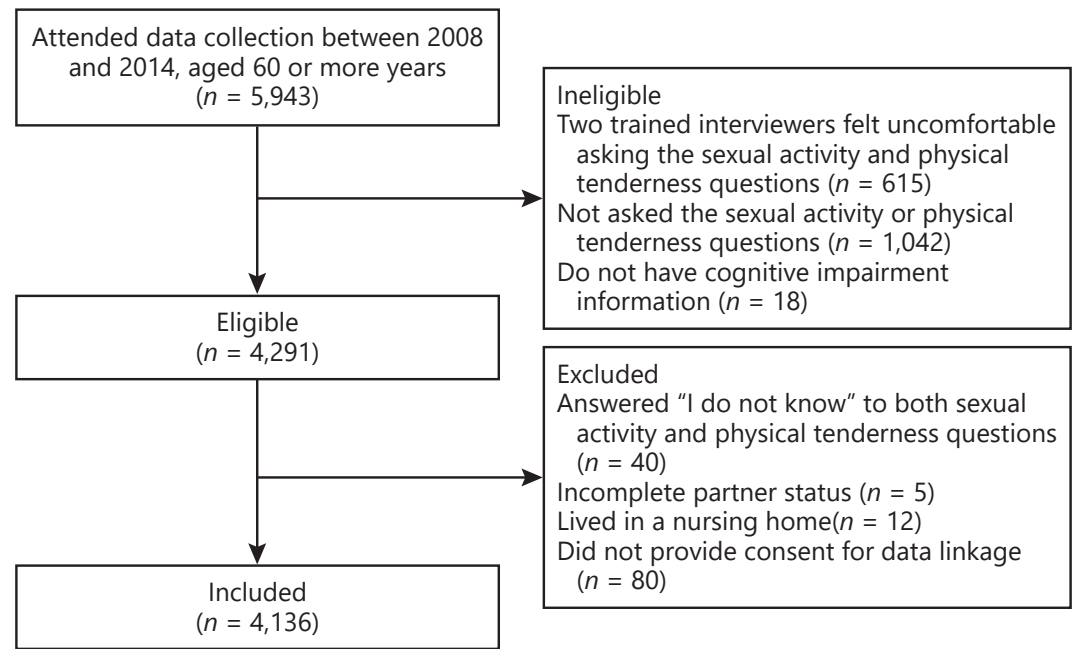

\section{Appendix 2}

Association between the act of being asked sexual activity questions by trained interviewers and demographic and health indicators ${ }^{a}$

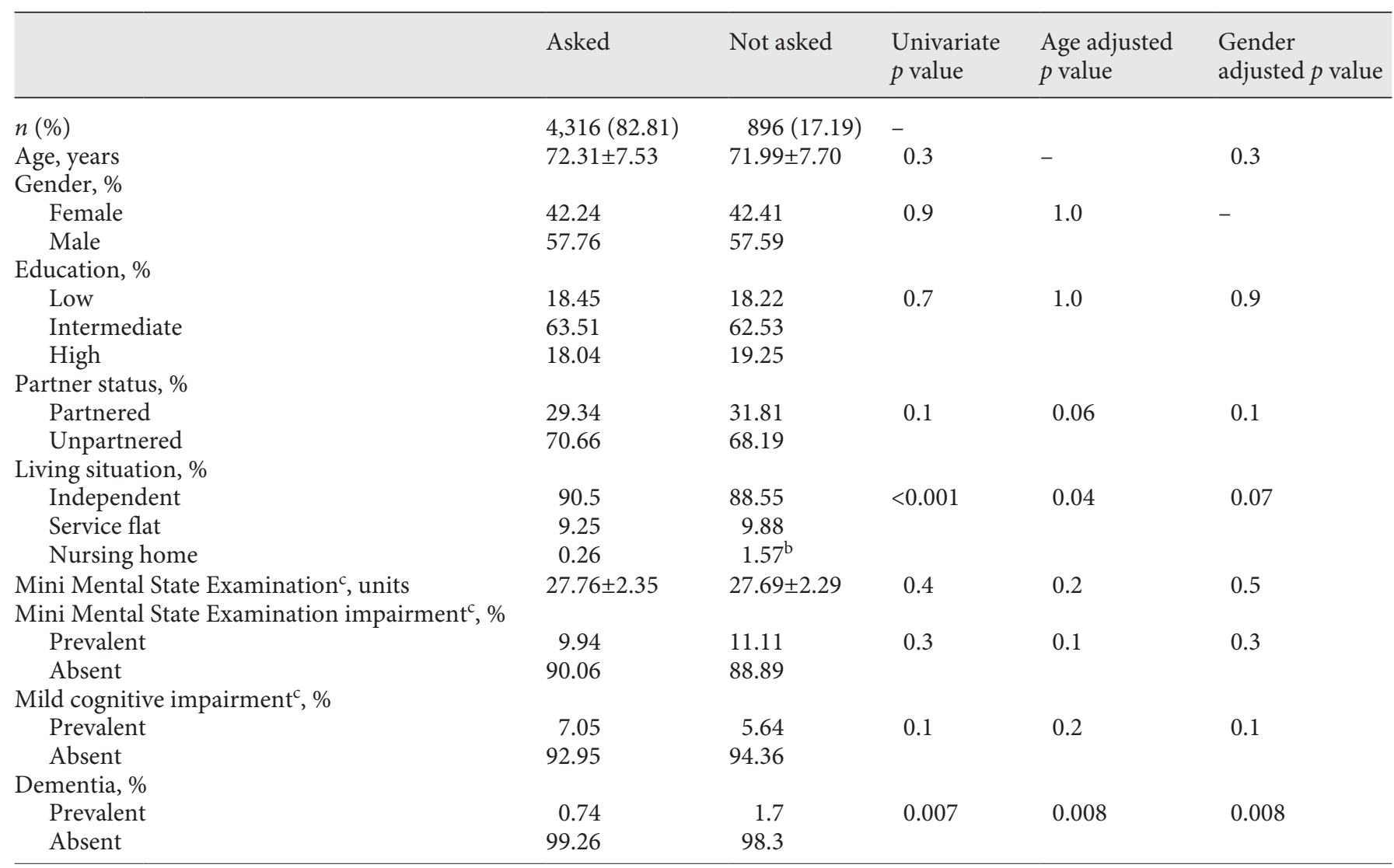

Cognitive Impairment, Sex and Tenderness 


\begin{tabular}{|c|c|c|c|c|c|}
\hline Activities of daily living, units & $0.47 \pm 0.51$ & $0.57 \pm 0.6$ & $<0.001$ & $<0.001$ & $<0.001$ \\
\hline \multicolumn{6}{|l|}{ Diabetes, \% } \\
\hline Prevalent & 15.36 & 13.85 & 0.3 & 0.4 & 0.3 \\
\hline Absent & 84.64 & 86.15 & & & \\
\hline Absent & 88.00 & 85.94 & & & \\
\hline \multicolumn{6}{|l|}{ Cancer, \% } \\
\hline Prevalent & 27.93 & 27.93 & 1.0 & 0.8 & 0.9 \\
\hline Absent & 72.07 & 72.07 & & & \\
\hline
\end{tabular}

a Participants were excluded from this table as they did not provide consent for data linkage $(n=24){ }^{\mathrm{b}}$ These participants are excluded from this study sample due to living in a nursing home, regardless of sexual activity questions not being asked. ${ }^{\mathrm{c}}$ Excludes participants with dementia.

\section{Appendix 3}

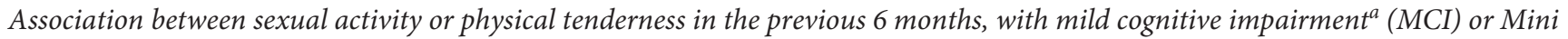
Mental State Examination (MMSE)

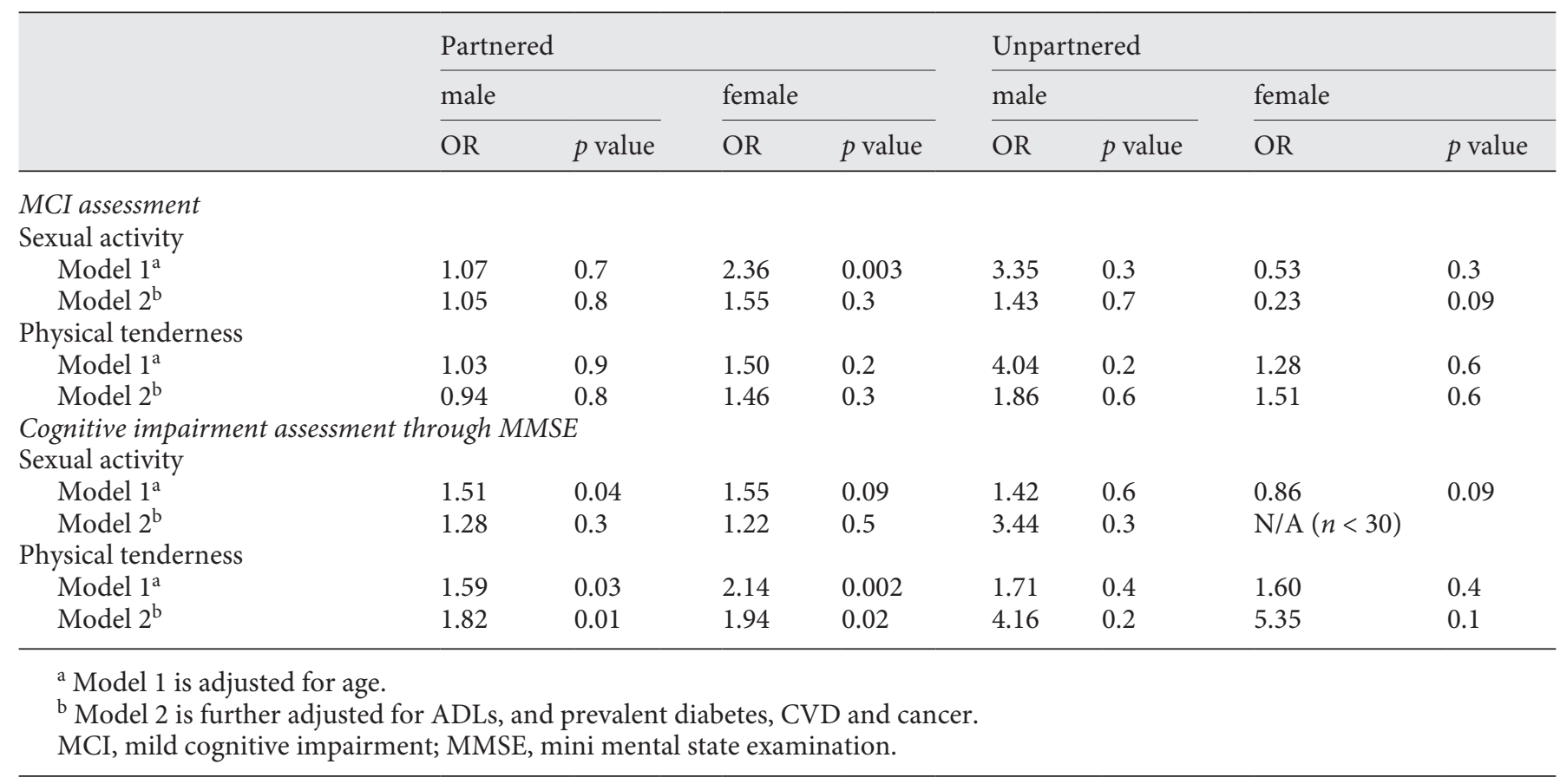




\section{References}

1 Karraker A, Delamater J, Schwartz CR: Sexual frequency decline from midlife to later life. J Gerontol B Psychol Sci Soc Sci 2011;66:502512.

2 Hooghe M: Is sexual well-being part of subjective well-being? An empirical analysis of Belgian (Flemish) survey data using an extended well-being scale. J Sex Res 2012;49: 264-273.

3 Rosen RC, Bachmann GA: Sexual well-being, happiness, and satisfaction, in women: the case for a new conceptual paradigm. J Sex Marital Ther 2008;34:291-297.

4 World Health Organization (WHO): Defining sexual health: Report of a technical consultation on sexual health: Sexual health document series. Geneva, WHO, 2002. www. who.int/reproductivehealth/publications/ sexual_health/defining_sexual_health.pdf (accessed February 19, 2018).

5 Freak-Poli R, Kirkman M, De Castro Lima G, Direk N, Franco OH, Tiemeier H: Sexual activity and physical tenderness in older adults: Cross-sectional prevalence and associated characteristics. J Sex Med 2017;14:918-927.

6 DeLamater J, Moorman S: Sexual behavior in later life. J Aging Health 2007;19:921-945.

7 Beckman N, Waern M, Ostling S, Sundh V, Skoog I: Determinants of sexual activity in four birth cohorts of Swedish 70-year-olds examined 1971-2001. J Sex Med 2014;11:401410.

8 Bach LE, Mortimer JA, VandeWeerd C, Corvin J: The association of physical and mental health with sexual activity in older adults in a retirement community. J Sex Med 2013;10: 2671-2678.

9 Lochlainn MN, Kenny RA: Sexual activity and aging. J Am Med Dir Assoc 2013;14:565-572.

10 DeLamater J, Sill M: Sexual desire in later life. J Sex Res 2005;42:138-149.

11 Jeong HC, Kim SU, Lee WC, Kim MT, Lee WK, Kim HY, Kim SY, Yang DY: Sexual behavior of the elderly in urban areas. World J Mens Health 2012;30:166-171.

12 Lee DM, Tajar A, Ravindrarajah R, Pye SR, O’Connor DB, Corona G, O'Connell M, Gielen E, Boonen S, Vanderschueren D, Pendleton N, Finn JD, Bartfai G, Casanueva FF, Forti G, Giwercman A, Han TS, Huhtaniemi IT, Kula K, Lean ME, Punab M, Wu FC, O’Neill TW: Frailty and sexual health in older European men. J Gerontol A Biol Sci 2013;68:837844.

13 Ganong K, Erik L: Intimacy and belonging the association between sexual activity and depression among older adults. Soc Ment Health 2011;1:153-172.

14 DeLamater J: Sexual expression in later life: a review and synthesis. J Sex Res 2012;49:125141.

15 Tower RB, Kasl SV: Gender, marital closeness, and depressive symptoms in elderly couples. J Gerontol B Psychol Sci Soc Sci 1996; 51:P115-P129.
16 Muller B, Nienaber CA, Reis O, Kropp P, Meyer W: Sexuality and affection among elderly German men and women in long-term relationships: results of a prospective population-based study. PLoS One 2014;9:e111404.

17 Wright H, Jenks RA: Sex on the brain! Associations between sexual activity and cognitive function in older age. Age Ageing 2016;45: 313-317.

18 Hartmans C, Comijs H, Jonker C: Cognitive functioning and its influence on sexual behavior in normal aging and dementia. Int J Geriatr Psychiatry 2014;29:441-446.

19 Wright H, Jenks RA, Demeyere N: Frequent sexual activity predicts specific cognitive abilities in older adults. J Gerontol B Psychol Sci Soc Sci 2017, Epub ahead of print.

20 Lee D, Tetley J: How long will I love you? Sex and intimacy in later life. London, The International Longevity Centre - UK, 2017. www. ilcuk.org.uk/images/uploads/publicationpdfs/ILC-UK_-_How_long_will_I_love_ you_1.pdf (accessed March 12, 2017).

21 Freak-Poli R, De Castro Lima G, Direk N, Jaspers L, Pitts M, Hofman A, Tiemeier H: Happiness, rather than depression, is associated with sexual behaviour in partnered older adults. Age Ageing 2017;46:101-107.

22 Ikram MA, Brusselle GGO, Murad SD, van Duijn CM, Franco OH, Goedegebure A, Klaver CCW, Nijsten TEC, Peeters RP, Stricker BH, Tiemeier H, Uitterlinden AG, Vernooij MW, Hofman A: The Rotterdam Study: 2018 update on objectives, design and main results. Eur J Epidemiol 2017;32:807-850.

23 OECD: Divided We Stand: Why Inequality Keeps Rising, OECD Publishing, 2011.

24 de Bruijn RF, Akoudad S, Cremers LG, Hofman A, Niessen WJ, van der Lugt A, Koudstaal PJ, Vernooij MW, Ikram MA: Determinants, MRI correlates, and prognosis of mild cognitive impairment: the Rotterdam Study. J Alzheimer's Dis 2014;42(suppl 3):S239-S249.

25 Roberts R, Knopman DS: Classification and epidemiology of MCI. Clin Geriatr Med 2013; 29:753-772.

26 Freak-Poli R, Mirza SS, Franco OH, Ikram MA, Hofman A, Tiemeier H: Positive affect is not associated with incidence of cardiovascular disease: a population-based study of older persons. Prev Med 2015;74:14-20.

27 de Bruijn RF, Bos MJ, Portegies ML, Hofman A, Franco OH, Koudstaal PJ, Ikram MA: The potential for prevention of dementia across two decades: the prospective, populationbased Rotterdam Study. BMC medicine 2015; 13:132.

28 Lawton MP, Brody EM: Assessment of older people: self-maintaining and instrumental activities of daily living. Gerontologist 1969;9: 179-186.

29 Fries JF, Spitz PW, Young DY: The dimensions of health outcomes: the health assessment questionnaire, disability and pain scales. J Rheumatol 1982;9:789-793.
30 Clayton D, Hill M: Statistical Models in Epidemiology. Oxford, New York Oxford University Press, 1993, p 273.

31 Leening MJ, Kavousi M, Heeringa J, van Rooij FJ, Verkroost-van Heemst J, Deckers JW, Mattace-Raso FU, Ziere G, Hofman A, Stricker BH, Witteman JC: Methods of data collection and definitions of cardiac outcomes in the Rotterdam Study. Eur J Epidemiol 2012; 27:173-185.

32 World Health Organization (WHO): Global Status Report on Noncommunicable Diseases, 2014. Geneva, WHO, 2014.

33 Leening MJ, Ferket BS, Steyerberg EW, Kavousi M, Deckers JW, Nieboer D, Heeringa J, Portegies ML, Hofman A, Ikram MA, Hunink MG, Franco OH, Stricker BH, Witteman JC, Roos-Hesselink JW: Sex differences in lifetime risk and first manifestation of cardiovascular disease: prospective population based cohort study. BMJ 2014;349:g5992.

34 Wasserstein RL, Lazar NA: The ASA's statement on p-values: context, process, and purpose. The American Statistician 2016;DOI: 10.1080/00031305.2016.1154108.

35 Dello Buono M, Zaghi P, Padoani W: Sexual feelings and sexual life in an Italian sample of 335 elderly 65-106-year-olds. Arch Gerontol and Geriatr 1998;26:155-162.

36 Padoani W, Dello Buono M, Marietta P, Scocco P, Zaghi PC, De Leo D: Influence of cognitive status on the sexual life of 352 elderly Italians aged 65-105 years. Gerontology 2000;46: 258-265.

37 Diamond L, Vahia I, Fellows I: Sexual satisfaction and successful cognitive and emotional aging in post-menopausal women. Am J Ger Psych 2009; 17:A136-A137.

38 Volkow ND, Gur RC, Wang GJ, Fowler JS, Moberg PJ, Ding YS, Hitzemann R, Smith G, Logan J: Association between decline in brain dopamine activity with age and cognitive and motor impairment in healthy individuals. Am J Psychiatry 1998;155:344-349.

39 Castanho TC, Moreira PS, Portugal-Nunes C, Novais A, Costa PS, Palha JA, Sousa N, Santos NC: The role of sex and sex-related hormones in cognition, mood and well-being in older men and women. Biol psychol 2014;103:158166

40 Haddad PM, Benbow SM: Sexual problems associated with Dementia: part 2. Aetiology, assessment and treatment 1993;8:631-637.

41 Rees PM, Fowler CJ, Maas CP: Sexual function in men and women with neurological disorders. Lancet 2007;369:512-525.

42 Hartmans C, Comijs H, Jonker C: The perception of sexuality in older adults and its relationship with cognitive functioning. Am J Geriatr Psychiatry 2015;23:243-252.

43 Galinsky AM, Waite LJ: Sexual activity and psychological health as mediators of the relationship between physical health and marital quality. J Gerontol B Psychol Sci Soc Sci 2014; 69:482-492. 
44 Richters J, de Visser RO, Badcock PB, Smith AM, Rissel C, Simpson JM, Grulich AE: Masturbation, paying for sex, and other sexual activities: the second Australian study of health and relationships. Sexual health 2014;11:461471.

45 Franco-Marina F, Garcia-Gonzalez JJ, Wagner-Echeagaray F, Gallo J, Ugalde O, Sanchez-Garcia S, Espinel-Bermudez C, JuarezCedillo T, Rodriguez MA, Garcia-Pena C: The Mini-mental State Examination revisited: ceiling and floor effects after score adjustment for educational level in an aging Mexican population. Int Psychogeriatr 2010;22: 72-81.

46 Rosen R, Altwein J, Boyle P, Kirby RS, Lukacs B, Meuleman E, O'Leary MP, Puppo P, Robertson C, Giuliano F: Lower urinary tract symptoms and male sexual dysfunc- tion: the multinational survey of the aging male (MSAM-7). Eur Urol 2003;44:637649.

47 Nicolosi A, Laumann EO, Glasser DB, Moreira ED Jr, Paik A, Gingell C: Sexual behavior and sexual dysfunctions after age 40 : the global study of sexual attitudes and behaviors. Urology 2004;64:991-997.

48 Lee DM, Nazroo J, O'Connor DB, Blake M, Pendleton N: Sexual health and well-being among older men and women in England: findings from the english longitudinal study of Ageing. Arch Sex Behav 2016;45:133144.

49 Wang V, Depp CA, Ceglowski J, Thompson WK, Rock D, Jeste DV: Sexual health and function in later life: a population-based study of 606 older adults with a partner. Am J Geriatr Psychiatry 2015;23:227-233.
50 Widmer E, Treas J, Newcomb R: Attitudes toward nonmarital sex in 24 countries. J Sex Res 1998;35:349-358.

51 Fileborn B, Lyons A, Hinchliff S, Brown G, Heywood W, Dow B, Malta S, Minichiello V: Improving the sexual lives of older Australians: perspectives from a qualitative study. Australas J Ageing 2017;36:E36-E42.

52 Carpenter L, Nathanson C, Kim Y: Sex after 40?: gender, ageism, and sexual partnering in midlife. J Aging Stud 2006;20:93-106.

53 Graf AS, Patrick JH: The influence of sexual attitudes on mid- to late-life sexual well-being: age, not gender, as a salient factor. Int J Aging Human Dev 2014;79:55-79.

54 Editors Michel J, Beattie B, Martin F, Walston J: Oxford Textbook of Geriatric Medicine, third edition. Glasgow, Oxford University Press, 2018 